In Situ

Revue des patrimoines

\section{In Situ}

Revue des patrimoines

27 | 2015

Le cheval et ses patrimoines (2e partie)

\title{
Le percheron à l'écomusée du Perche (Orne)
}

\section{Évelyne Wander}

\section{OpenEdition}

Journals

Édition électronique

URL : http://journals.openedition.org/insitu/11935

DOI : 10.4000/insitu. 11935

ISSN : 1630-7305

\section{Éditeur}

Ministère de la culture

\section{Référence électronique}

Évelyne Wander, «Le percheron à l'écomusée du Perche (Orne) », In Situ [En ligne], 27 | 2015, mis en ligne le 03 novembre 2015, consulté le 20 avril 2019. URL : http://journals.openedition.org/ insitu/11935; DOI : 10.4000/insitu.11935

Ce document a été généré automatiquement le 20 avril 2019.

\section{(c) $($ ) $(9)$}

In Situ Revues des patrimoines est mis à disposition selon les termes de la licence Creative Commons Attribution - Pas d'Utilisation Commerciale - Pas de Modification 4.0 International. 


\title{
Le percheron à l'écomusée du Perche (Orne)
}

\author{
Évelyne Wander
}

1 Adopté par notre établissement, en 2000, le concept d'écomusée nous a permis d'appréhender un patrimoine complexe, celui de l'espace rural, dont les pouvoirs publics espèrent de sa valorisation un ferment de développement. L'homme et son milieu sont au centre de toutes les actions de l'écomusée en matière de conservation et de médiation.

2 Cette approche du patrimoine et du monde contemporain nous a permis de renouveler la programmation culturelle et nos missions de conservation. La collection du musée est classée et contrôlée depuis 1985, elle appartient au conseil général de l'Orne.

3 La présentation de l'exposition permanente, existante depuis 1985, est celle d'un musée d'art et de tradition populaire (dont Georges-Henri Rivière regrettait lui-même la terminologie malheureuse). Une grande place avait été faite au thème du cheval percheron au sein de la présentation permanente, le concepteur a regroupé des reconstitutions d'atelier : bourrelier, charron, maréchal-ferrant, afin de mettre en valeur les liens existants entre artisanat et cheval. Le Perche a été une zone d'élevage équin renommée. Ce territoire a donné son nom à un cheval de trait réputé pour sa robustesse et son calme : le percheron.

4 Nous examinerons au long de cet article si le fait de disposer d'une collection rend compte du patrimoine lié au cheval et sert la cause d'un patrimoine vivant, en l'occurrence celle d'une race de trait qui a dû sa sauvegarde à son utilisation bouchère. Un beau cas d'ironie du sort!

5 Lors de la création du musée, dans les années 1970, les concepteurs avaient privilégié le cheval percheron, porteur d'une image emblématique du territoire. Ce choix a éclipsé d'autres élevages du parcours muséographique. Ce regard sur le passé perdure au point que certains éleveurs de chevaux percherons niaient, encore récemment, jusqu'à la présence d'autres animaux de traction dans le Perche au début du xxe siècle. 
6 L'élevage bovin, encore très présent sur le territoire à cette période (quota laitier 1984), ne présentait pas de légitimité patrimoniale. Le deuil de la séparation du cheval au profit $\mathrm{du}$ tracteur sonne comme la disparition d'un compagnon de travail (formant le couple percheron-percheron).On demandait une résilience, une réparation. Le musée a, en quelque sorte, rempli ce rôle, enjoint de jouer la beauté du mort (où le populaire devient attractif une fois éteint). Certaines lectures d'Octave Mirbeau (1848-1917), qui a vécu à Rémalard (commune du Perche) ne laissent pas penser cependant à une affection toute unilatérale vers ce cheval qui fut exporté massivement aux États-Unis (1840-1914) afin de conquérir puis de cultiver les grandes plaines de l'Ouest américain ou de tracter des omnibus...

7 Le cheval percheron a été l'objet d'un commerce important également vers Paris. Le développement de la traction motorisée pour le transport des marchandises et des personnes a eu raison d'une économie locale très hiérarchisée autour de grands propriétaires, véritables entrepreneurs de l'élevage (familles Aveline, Chouasnard, Chapelle...). Si certains noms restent attachés au monde du cheval percheron, le réseau des petits éleveurs, des utilisateurs locaux, d'un salariat agricole important, d'un artisanat local et d'un artisanat de haut niveau (Maison Chalopin, sellier à Nogent-LeRotrou) a disparu aujourd'hui.

8 De nombreux bâtiments ruraux de l'espace percheron portent encore témoignage de cette épopée. L'édifice le plus abouti et demeuré en bon état de conservation reste la Ferme neuve à Dorceau, ancienne exploitation construite par Josèphe Aveline (1881-1958). La question de ce patrimoine bâti spécifique, à l'instar, pour sa plus grande majorité, du patrimoine agricole en France, reste aux mains de propriétaires privés. Les haras nationaux, la bergerie de Rambouillet, certains musées installés dans des maisons de maître... font figure d'exception car elles relèvent du patrimoine prestigieux. Ruinés dans le pire des cas, transformés au rythme des évolutions techniques de l'agriculture, ou changeant radicalement de destination, les bâtiments agricoles disparaissent ou sont profondément transformés. Nous ne faisons pas différemment de nos devanciers en la matière, il n'est de monument historique ou de ville qui n'attestent de ces pratiques d'éradication ou de profonds remaniements architecturaux. Cependant on s'interrogera sur le devenir du patrimoine agricole et industriel qui ne représente que l'un des maillons d'une chaîne économique complexe.

9 Fort heureusement, pour ce qui nous concerne, l'acquisition, en 1993, de l'ensemble prieural de Sainte-Gauburge, comprenant les bâtiments conventuels et agricoles, a permis de concevoir un projet muséographique plus ambitieux, en particulier pour les représentations de l'élevage et notamment pour celui du cheval percheron. En effet, les écuries, si elles ont perdu leurs bas-flancs et une partie des râteliers, sont encore en bon état. Elles ont été construites dans le dernier quart du $\mathrm{XIX}^{\mathrm{e}}$ avec un grand soin, en attestent et les abreuvoirs et le pavage en granit régional (Alençon). Un logement de palefrenier avec fonction de sellerie est attenant. Il est aujourd'hui meublé avec des pièces de notre collection.

10 Si l'on considère les éléments dont nous disposons en tant qu'écomusée afin de rendre compte de l'histoire (celle du cheval urbain restant encore peu représentée) du cheval agricole :

11 - une collection importante liée à l'artisanat, à la traction pour outils agricoles, des récompenses de concours agricoles, des documents publicitaires, quelques œuvres, des 
éléments documentaires, des productions audiovisuelles. Le chantier des collections projeté est toujours dans l'attente de son financement,

12 - une écurie modestement scénographiée et encore dans l'attente d'une restauration, aussi inquiétante que l'absence de celle-ci,

13 - un espace dédié au sein du musée où figurent quelques œuvres, les boutiques reconstituées de l'artisanat équin : maréchal-ferrant, bourrelier, charron.

14 Sommes-nous mieux lotis à ce stade qu'un muséum devant rendre compte de l'habitat du diplodocus?

15 L'arbitrage, que doit rendre tout concepteur de musée, repose, dans le cas de la création du musée des Arts et Traditions du Perche, sur l'accumulation de preuves matérielles, d'objets très nombreux nécessaires à la traction et à la mécanisation dans les domaines très divers du transport et de l'agriculture (fig. 1). L'aspect matériel des choses ne peut tenir lieu de justification muséographique. Le patrimoine immatériel a souvent fait les frais de ce respect pour l'objet dans de petits établissements qui ne pouvaient mener de front l'organisation de la conservation matérielle, l'enquête ethnographique et la recherche historique. Enfin, concernant le cheval percheron, nous sommes face à un autre dilemme, celui d'un patrimoine vivant difficile à placer derrière une vitrine.

Figure 1

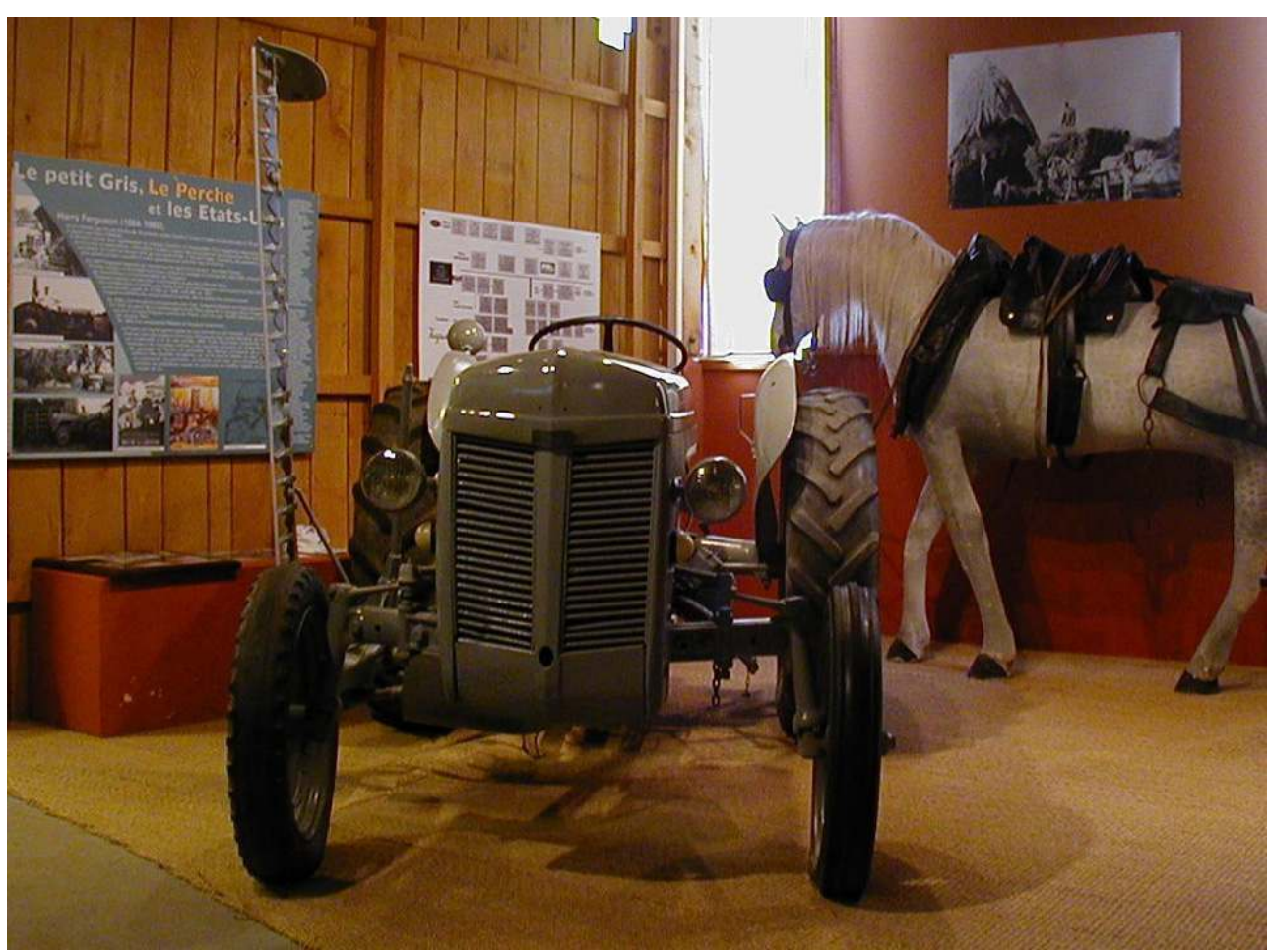

Écomusée du Perche (Saint-Cyr-la-Rosière, Orne). Au premier plan tracteur Massey-Ferguson, au second plan figure de comice représentant un cheval percheron.

PHOT. WANDER, ÉVELYNE. ( C ÉVELYNE WANDER.

Les missions de conservation du patrimoine du musée ont $\mathrm{pu}$, dans notre cas, être prolongées par l'ouverture sur le territoire permis par le concept d'écomusée.

Nous nous sommes posé la question d'acquérir un cheval. Était-ce notre rôle ? En tout état de cause, cela ne relevait pas de nos compétences. Le seul cheval « administratif » viable 
étant celui des haras nationaux, nous avons renoncé pour une autre formule, en cohérence avec la philosophie de l'écomusée. Nous avons mis au point un programme plus fédérateur pour le territoire et garantissant la conservation de savoir-faire et d'une culture du cheval percheron.

Ateliers pédagogiques, stages, journée à thème, circuits touristiques ont permis de travailler en coopération avec des éleveurs aux statuts fort différents. Nous avons développé grâce à une collègue, Évelyne Morin, qui s'est spécialisée dans le domaine de l'élevage du percheron, un réseau d'éleveurs amateurs et professionnels et un partenariat de plus en plus construit avec la Société hippique percheronne.

Non seulement nous participons au développement économique de notre territoire mais nous œuvrons, aussi, à la diffusion de connaissances auprès des éleveurs et des publics. Le travail avec le jeune public contribue au processus de conservation en éveillant les jeunes citoyens à la connaissance d'un patrimoine fragile parce que vivant.

20 La journée à thème, intitulée "Fête du cheval percheron", est aujourd'hui la manifestation la plus importante de notre établissement. Nous accueillons sur notre site 3500 visiteurs (fréquentation annuelle 26300 visiteurs) (fig. 2). Le contenu repose pour une partie sur des présentations patrimoniales (harnachement, voitures anciennes, machines agricoles telles la trépigneuse) appartenant à la collection du musée ou à d'autres partenaires tel le COMPA (Musée du machinisme agricole-Chartres) mais également à des collectionneurs - éleveurs qui conservent dans une perspective dynamique des matériels anciens.

Figure 2

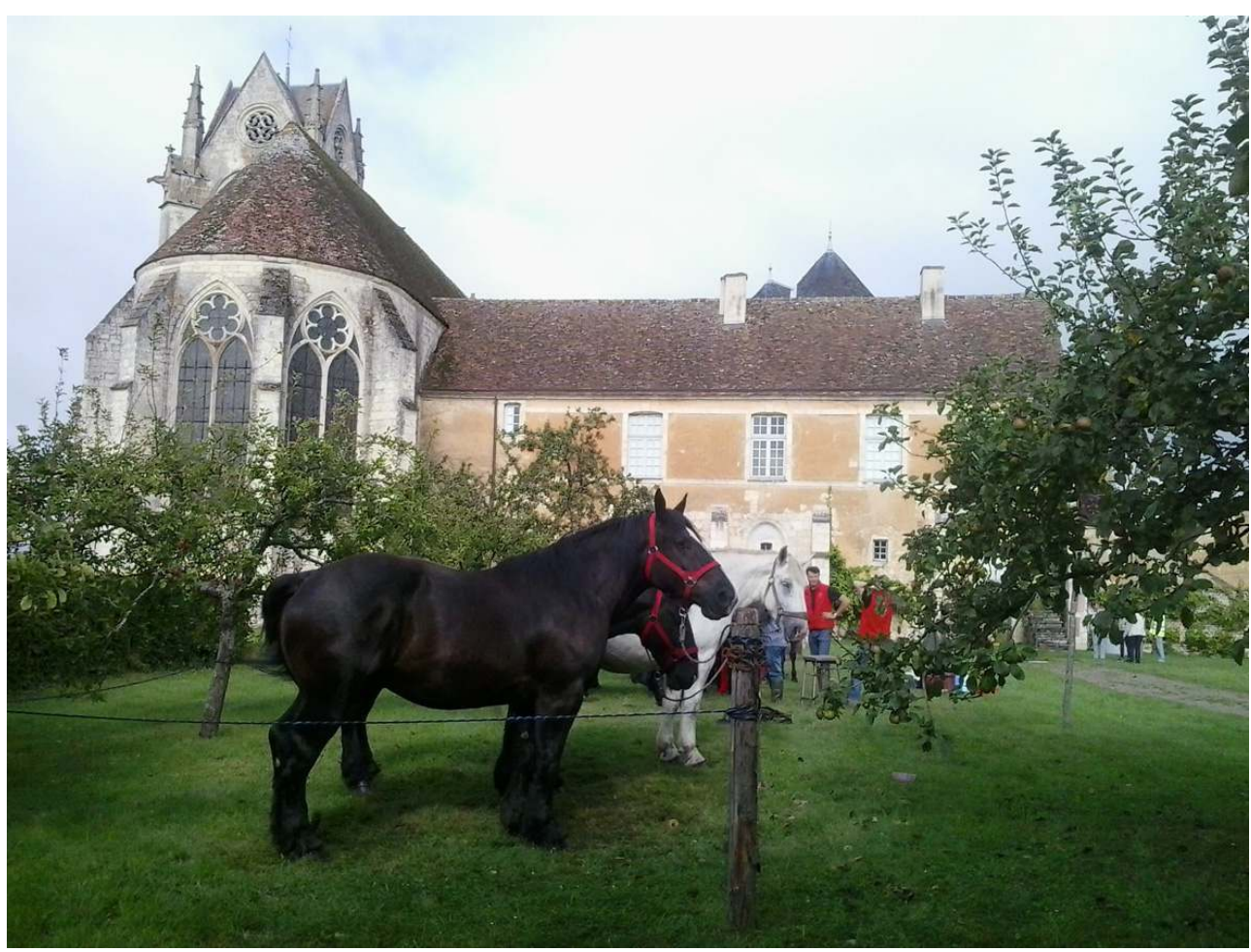

Écomusée du Perche (Saint-Cyr-la-Rosière, Orne). Présentation de percherons dans le verger du prieuré de Sainte-Gauburge.

PHOT. WANDER, ÉVELYNE. C ÉVELYNE WANDER. 
Cependant, pour une autre partie, la diffusion de connaissances propres à l'élevage est un temps très représentatif de notre action. La préparation du cheval (tressage, sabots, harnachement, tenue du meneur) est conduite comme un atelier pédagogique. La perspective est de rappeler les critères de présentation lors de concours. Les éleveurs professionnels ont un rôle de conseil auprès des éleveurs amateurs, lesquels participent pleinement de l'avenir de la race percheronne. La participation d'un vétérinaire, d'un dentiste équin contribue également à la diffusion de conseils de santé pour l'ensemble des éleveurs. Une cinquantaine de chevaux sont présents sur le terrain, ce qui est assez significatif de l'impact de ce travail, mené dans une ambiance festive.

Un aspect primordial de cette manifestation est l'ouverture sur les utilisations actuelles du cheval: débardage, agriculture biologique, traction en milieu urbain, techniques agricoles transférables vers les pays émergents.

Une autre fonction de cette manifestation contribuant à sa réussite est la pédagogie de projet. Le milieu des éleveurs amateurs et professionnels se caractérise par un comportement assez individualiste, renforcé par la culture du concours et la question de la vente des produits de l'élevage. La préparation de cette manifestation menée par ma collègue se fait très en amont avec la participation des intervenants. L'entraide, la mise en commun vers un but précis, lesquelles semblent des évidences, ont été en l'occurrence le résultat d'un patient travail.

On peut légitimement se poser la question du bien-fondé de cette politique quand d'autres structures - le parc naturel régional du Perche et la Société hippique percheronne - travaillent également au rayonnement à la valorisation de cette race.

Musée d'ethnographie augmenté du concept d'écomusée, nous travaillons sur un patrimoine instable, celui du champ culturel du cheval de trait sur un territoire qui est le berceau de la race percheronne, aux côtés d'autres acteurs.

En effet, le parc du Perche développe des actions sur une filière d'élevage basées sur deux axes : le monde agricole et le tourisme.

Quant à elle, la Société hippique percheronne, gardienne du stud-book, tient un rôle de représentation des éleveurs et de développement de la race qui a connu beaucoup d'évolutions en fonction des besoins de la société, passant du travail à l'alimentation, des loisirs (et aujourd'hui) au développement durable.

Si la démonstration du bien-fondé au regard de la complémentarité territoriale semble patente, nous pouvons revenir sur la question de la durabilité de cette action au service de la conservation du patrimoine équin composé de trois axes :

- la conservation du vivant

- la conservation des artefacts

- et la conservation du patrimoine immatériel.

Dernier axe qui est primordial pour la préservation des deux premiers par la conscience qu'il donne de l'intérêt même de la préservation de la race, par les renseignements recueillis pour documenter les collections matérielles.

Afin de consolider cette politique, nous avons également présenté des expositions, «Petite histoire du cheval percheron» et "Mode équine», qui ont donné lieu à la publication de deux catalogues. Trois films documentaires ont été réalisés, l'un sur l'élevage, l'autre sur la bourrellerie et un dernier sur le charronnage. Ce travail, entrepris 
à la fin des années 1990, est néanmoins à actualiser afin d'appréhender les nouvelles réalités.

34 Cependant, il nous reste beaucoup à faire pour la conservation des artefacts conservés dans nos réserves. Le chantier des collections envisagé attend son financement, la restauration des engins agricoles, des charrettes est encore à mener. Nous avions, dans les années 1990, restauré des séries d'harnachement sur notre seule initiative, en les confiant à un bourrelier installé à quelques kilomètres de l'écomusée. Les sommes engagées permettaient cette autonomie. La méthodologie de cette action n'est pas envisageable pour des séries d'objets de grande dimension. Nous avions pu mener un chantier de peinture pour certains, en embauchant un peintre qui a appliqué des peintures dont nous tenions les recettes d'un charron retraité.

Menant ces actions afin de préserver ce patrimoine, nous avons du même coup contourné les règles de la restauration des Musées de France, les protocoles consacrés nous engageant à utiliser les services d'un restaurateur. Nous nous posons la question de ces mesures, étant donné que cette exigence suppose l'intervention d'un restaurateur agréé. Dans cette perspective, les coûts sont démultipliés en raison de l'éloignement des ateliers ; il faut aussi souligner la rareté de ce type de compétences.

Dans l'esprit des actions que nous menons, il nous paraîtrait adéquat de travailler avec les rares artisans charrons qui travaillent encore en région en suivant un protocole rapproché de la direction des Patrimoines (fig. 3). De plus, notons que dans une société qui connaît une crise de l'emploi, chaque niche économique est aussi à encourager.

Figure 3

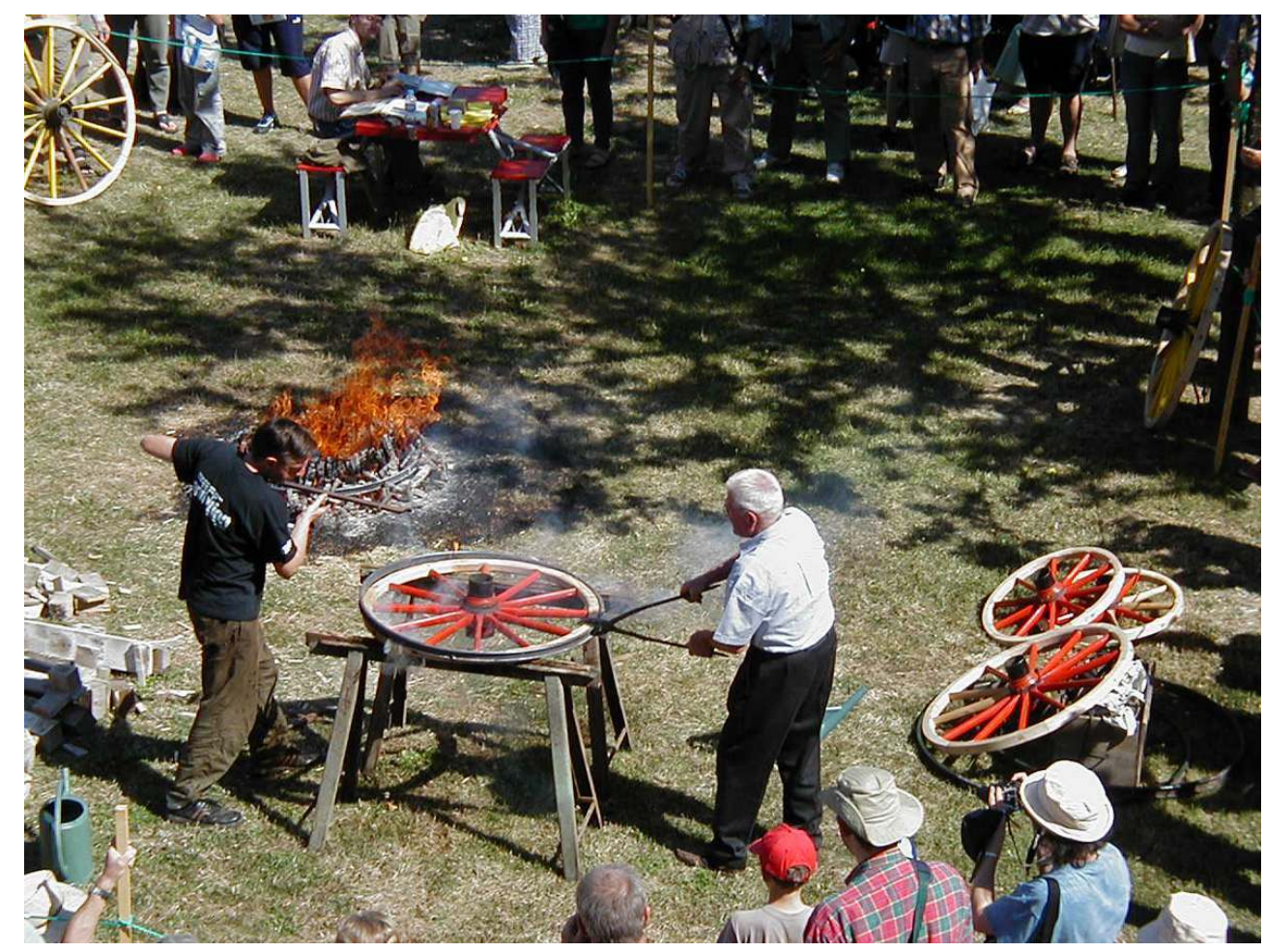

Écomusée du Perche (Saint-Cyr-la-Rosière, Orne). Atelier de charron, châtrage d'une roue lors d'une manifestation dédiée au cheval percheron.

PHOT. WANDER, ÉVELYNE. (C) ÉVELYNE WANDER. 
37

Nos collections agricoles ont souffert des premières conditions de collecte à l'origine du musée créé par une association non soutenue par des compétences professionnelles : un collectage massif peu renseigné et dont le résultat fut entreposé aux abords mêmes de l'établissement.

Le classement de la collection n'a pas fait évoluer les conditions de conservation, seul l'inventaire a pu être mené. Nous avons pu, depuis 1993, commencer à organiser la préservation des ces gros matériels. Cependant, nos réserves sont loin d'être suffisantes en ce qui concerne l'équipement et la place. Quand le chantier pourra être conduit - la date n'en est pas connue aujourd'hui - nous serons amenés à détruire une partie de ces collections après les précautions d'usage : photographies, levée de plans afin de préparer la création de maquettes ou l'acquisition de matériel identique.

Le manque de place et l'état de délabrement avancé de certains objets ont eu raison des missions qui nous avaient été confiées et de la confiance placée par les donateurs dans l'institution muséale. Je qualifierai cette situation de petit désastre.

40

De ces matériels qui ont été fabriqués en grand nombre, combien en reste-t-il aujourd'hui dans les collections publiques? Le COMPA, musée du machinisme agricole, est un exemple pour la conservation de ce type de collections par les moyens qu'il peut y consacrer et l'ouverture d'un atelier de restauration en interne. Cet établissement fait figure d'exception. Pour avoir participé aux réunions du groupe agriculture du réseau des musées de Basse-Normandie, nous avons pu faire le constat que cette situation inquiétante des collections agricoles et par conséquent des matériels à traction équestre est partagée par un grand nombre d'établissements.

41 Force est de constater que les collectionneurs privés conservent mieux ce patrimoine qui rend compte d'un univers technologique révolu, celui de l'énergie animale au service de la production agricole et industrielle. C'est pourquoi j'ai accepté votre invitation à témoigner.

Nous avons essayé de répondre à l'intitulé du colloque « Le cheval et ses patrimoines » en tentant de démontrer que des actions sont possibles pour la valorisation de ce patrimoine au service des territoires ruraux mais qu'il est, aujourd'hui, urgent et nécessaire de penser à un plan de sauvegarde des engins et moyens de transport à traction équine. Des protocoles de restauration, des mutualisations d'espaces de réserves, des groupes de travail nationaux font partie des solutions possibles afin de conserver les aspects les moins prestigieux du patrimoine équestre mais indispensables à la compréhension de l'histoire des techniques précédant l'ultra-motorisation du monde économique occidental.

43 À l'heure où notre société se pose la question de la finitude des ressources naturelles, concernant la conservation des collections du patrimoine équestre, il n'est pas anachronique de la penser comme une question d'actualité. 


\section{RÉSUMÉS}

Adopté par notre établissement, en 2000, le concept d'écomusée nous a permis de mieux appréhender le patrimoine rural du Perche, complexe par sa diversité, son format souvent et surtout sa relation au temps présent. Le patrimoine équin illustre parfaitement cet état de fait. Le cheval percheron tient une large place dans nos collections à l'instar de la charge symbolique que représente cet animal. Ce laissé pour compte de la révolution agricole de la seconde moitié du XX ${ }^{e}$ siècle est tout à la fois un objet de remords, de fascination, d'espoir de revival, une figure métonymique de l'agriculture semi-autarcique. Au cœur d'un grand nombre d'actions de médiation de l'écomusée, les artefacts liés à ce cheval de trait connu mondialement servent la cause d'un patrimoine vivant au service d'un territoire rural en pleine mutation. Cependant, il est aujourd'hui, urgent et nécessaire de penser à un plan de sauvegarde des engins et moyens de transport à traction équine. Des protocoles de restauration, des mutualisations d'espace de réserves, des groupes de travail nationaux font partie des solutions possibles afin de conserver des aspects moins prestigieux du patrimoine équestre mais indispensables à la compréhension de l'histoire des techniques précédant l'ultra motorisation du monde économique occidental. À l'heure où notre société se pose la question de la finitude des ressources naturelles, concernant la conservation des collections du patrimoine équestre, il n'est pas anachronique de la penser comme une source d'innovation pour l'environnement.

The Percheron draft horse at the Perche Ecomusée (Orne). The Perche museum became an ecomuseum in 2000. A new approach tried to take into account the extremely diverse rural heritage of the region, of considerable complexity in its relations with the present day. The horse heritage is a good example of this complexity. The Percheron horse occupies a large place in the museum's collections, in keeping with the symbolic load that this animal now carries. Left aside by the agricultural revolution of the second half of the twentieth century, the Percheron is an object of remorse, of fascination, of hopes for a revival, a figure symbolising the possibility of semi-self-sufficient agriculture. The Percheron is at the heart of many of the museum's interpretative initiatives and the artefacts associated with this horse of world-wide fame can serve the cause of a living heritage within a rural world undergoing profound changes. It is vital today to undertake a programme to safeguard and preserve the vehicles and techniques of horsedrawn transport. Protocols of restoration, the sharing of reserve spaces in museums, the setting up of nation-wide research groups are amongst the possible solutions in order to conserve the less prestigious aspects of the horse-related heritage, but vital for an understanding of the history of techniques before the ultra-motorisation of the western world. At a time when society begins to think more seriously about how the planet's natural resources are not infinite, it is perhaps not anachronistic to see the preservation of the horse-related heritage as an investment, a source of innovation for the environment.

\section{INDEX}

Mots-clés : écomusée, cheval de trait, cheval percheron, perche, ruralité

Keywords : ecomuseum, workhorse, Percheron, Perche region, rurality 
AUTEUR

\section{ÉVELYNE WANDER}

ethnologue, directeur et responsable scientifique de l'Écomusée du Perche direction@ecomuseeduperche.fr 